\title{
Impact of laparoscopic ovarian drilling on the pregnancy rate in clomiphene-resistant polycystic ovarian syndrome patients undergoing in vitro fertilization: randomized controlled trial
}

\author{
Adel M. Nada*, Hala Abdelwahab, Hala Nabil and Reham A. Mohsen
}

\begin{abstract}
Background: The main objective of this randomized controlled trial was to study the impact of LOD on the pregnancy rate after ICSI-ET in PCOS.

Results: The study was conducted in Egypt in the period 2015-2017 and included 212 clomiphene-resistant PCOS patients, with at least 1-year infertility. The study group was the drilling group who underwent LOD and then ICSI$E T$, while the control group did not undergo LOD but directly proceeded to ICSI-ET. The primary outcome was the clinical pregnancy rate per ET cycle. The baseline characteristics and hormonal profiles were comparable $(p>0.05)$ between the two groups. Ovarian stimulation days were $(p<0.001)$ higher in the drilling group. Endometrial thickness, estradiol at triggering day, and the number of oocytes retrieved were $(p<0.001)$ lower in the drilling group. The numbers of embryos transferred were not different $(p>0.05)$. The clinical pregnancy rate per ET cycle was higher in the drilling group (51\%) than in the control group (37\%) $(p=0.046)$. Multiple pregnancies were not significantly $(p=0.265)$ different between groups. The rate of OHSS was $(p=0.046)$ higher in the control group. Coasting was $(p<0.001)$ higher in the control group $(18 \%)$ compared to the drilling group $(2 \%)$.
\end{abstract}

Conclusion: Laparoscopic ovarian drilling for PCOS patients before ICSI-ET improves the clinical pregnancy rate with a reduction of OHSS.

Trial registration: Clinical Trial Registration: Pan African Clinical Trials Registry (PACTR), PACTR201604001567272, 5 April 2016.

Keywords: PCOS, clomiphene citrate resistant infertility, ICSI, Laparoscopy, Laparoscopic Ovarian Drilling (LOD)

\section{Key message}

The laparoscopic ovarian drilling for PCOS patients with clomiphene-resistant infertility improves ICSI-ET outcome, as it increases the clinical pregnancy rate with the reduction of the incidence of OHSS.

\section{Background}

Polycystic ovarian syndrome (PCOS) is the most prevalent endocrine disorder during the reproductive period

\footnotetext{
* Correspondence: dr.adel.nada@gmail.com

Department of Gynecology and Obstetrics, Faculty of Medicine, Cairo University, Al-Manyal St. Giza, Cairo 11433, Egypt
}

[1]. According to the published literature, its prevalence range from 5 to $10 \%$ among women of this period [2-5]. However, when the European Society for Human Reproduction and Embryology/American Society for Reproductive Medicine criteria is used, the prevalence is as high as $15-20 \%$ [6]. The most critical impact of PCOS resulted in ovulation dysfunction [1-6].

The management of PCOS is dependent upon the symptoms presented: androgen-related symptoms, menstruationrelated symptoms, and infertility [7]. In PCOS patients with infertility, modification of lifestyle is the first-line approach in addition to the clomiphene-citrate (CC) and other selective 
estrogen receptor modulators (SERMs) [3]. However, CC resistance was seen in $20 \%$ of patients with unsuccessful induction of ovulation [8]. The second-line of treatment is the gonadotropin therapy with its higher risks of ovarian hyper-stimulation syndrome (OHSS) and the consequent multiple pregnancies [9].

Laparoscopic ovarian drilling (LOD), using laser or electrocautery to make 4-10 holes in ovarian surface and stroma, is indicated for the management of infertility in patients with PCOS resistant to CC (Thessaloniki ESHRE/ASRM, 2008). One study showed the reestablishment of ovulatory menstrual cycles in the majority of cases and pregnancy in more than 50\% [10]. However, there is a lack of consensus on the effectiveness of LOD for ovulation induction [7, 11]. Also, the addition of LOD to gonadotropins for ovulation induction is advocated as a second-line option by the European Society of Human Reproduction and Embryology and the American Society for Reproductive Medicine (ESHRE/ASRM) [12].

A third-line therapy for those patients with CCresistant infertility is the intra-cytoplasmic sperm injection/embryo transfer (ICSI-ET) [13].

However, the effect of LOD on ICSI-ET outcomes in PCOS patients is still controversial. Thus, the rationale intended for this parallel randomized controlled study was to study the impact of LOD on the pregnancy rates of ICSI-ET in PCOS patients with clomiphene-resistant infertility.

\section{Methods}

This parallel randomized controlled trial was conducted in the Obstetrics \& Gynecology Department, Cairo University Hospital, and in Obstetrics and Gynecology Department, Beni-Suif University Hospital, during the period from January 2015 to January 2017. This study followed the principles of the Declaration of Helsinki and in accordance with the Medical Research Involving Human Subjects Act (WMO) and was approved by the medical ethical review committee of Cairo University on December 14, 2014, with registration number no: OG-514-12-2014. The purpose of this study was clearly explained in the Arabic language to all subjects before their enrollment to the study, and an informed consent form was signed by and obtained from all of those enrolled.

We recruited patients attending infertility unit in the two centers with age between 20 and 35 years old, history of at least 1-year infertility, and no response to CC for at least three cycles. Patients diagnosed with PCOS according to Rotterdam consensus. To consider a patient as a PCOS patient, two criteria out of three should be present. Criteria are oligo/or anovulation, hyperandrogenism, and polycystic ovarian morphology by transvaginal ultrasound.
Exclusion criteria included the following: women with any other cause of oligomenorrhea and hyperandrogenism were excluded. Furthermore, patients with the following criteria were excluded: history of previous ICSI-ET, chronic diseases such as thyroid disorders and diabetes mellitus; women who received hormones or drugs for major medical diseases; women who presented ovarian tumors; patients who underwent LOD outside our institute; severe endometriosis, uterine anomalies, or hydrosalpinx documented by hystero-salpingeography, ultrasound, or hysteroscopy and infertility due to severe male factor (azoospermia).

\section{Randomization and blinding}

For the allocation of the participants, a computergenerated list of random numbers was used. Block randomization with a block size of 4 was used with 1:1 ratio of drilling and control group. The allocation sequence was concealed from the researchers enrolling and assessing participants. The study was assessorblinded.

Participants were randomly allocated to the study group and the control group. The study group underwent LOD and then proceeded into one ICSI-ET cycle. The control group proceeded directly into one ICSI-ET cycle without LOD. Neither the researcher allocating the participants nor the assessing person knew the decoding of the groups in its relation to the allocation sequence. However, the physicians who did the ICSI-ET were not blinded.

\section{Procedures: Laparoscopic ovarian drilling}

Laparoscopic ovarian drilling (LOD) was done in the two institutes using the standard routine procedure done in Cairo University Hospitals under general anesthesia. Inflation was made by $\mathrm{CO}_{2}$ up to $14 \mathrm{mmHg}$. Three ports were done one at the umbilicus for the camera, and two side ports for manipulating and holding the ovary. Fiftywatt current (coagulation mode) was used making four holes each lasting $4 \mathrm{~s}$ at a depth of 3-4 $\mathrm{mm}$ to both ovaries. The time interval between the drilling and IVF was $1-3$ cycles.

\section{Procedures: ICSI}

After the explanation of the whole procedure and before starting the ovarian stimulation, fasting blood samples from all eligible women, for basal early follicular phase serum AMH, FSH, LH, prolactin, TSH, and preceding ICSI cycle. All patients underwent baseline transvaginal sonography on day 2 of the menstrual cycle to check for antral follicle count (AFC) and endometrial thickness and to rule out the presence of an ovarian cyst.

AFC was done at day 2 of the cycle next to that of drilling counting all follicles from $4-9 \mathrm{~mm}$. Ovarian 
stimulation was started on day 2 of the cycle by injection of gonadotropins (Merional-IBSA, Switzerland) (150300 IU daily) according to AFC, anti-Mullerian hormone (AMH), and BMI; and the dose is adjusted according to follicular development. We continued the same dose if there was an adequate response (fixed dosage protocol). In some patients, we needed to increase the dose (stepup) or decrease the dose (step-down dosage protocol). Cetrorelix acetate (Cetrotide-Merck-Serono, Germany) $0.25 \mathrm{mg} \mathrm{s} / \mathrm{c}$ treatment was started when the leading follicle reached a diameter of $14 \mathrm{~mm}$ and/or the estradiol levels were $>400 \mathrm{pg} / \mathrm{ml}$. Treatment with merional and antagonist was continued till the day of the final oocyte maturation trigger. When three or more follicles of size $18 \mathrm{~mm}$ or more were seen, the final oocyte maturation trigger was given with Choriomon (IBSA, Switzerland) injection HCG 5000 IU intramuscular. We did not trigger ovulation by $\mathrm{Gn} \mathrm{RH}$ agonist as it may reduce the clinical pregnancy rate. Transvaginal ultrasound-guided oocyte aspiration (OPU) was performed approximately 35-36 h after hCG injection under general anesthesia. Conventional ICSI was performed. Fertilization was defined as the presence of pronuclei $16-18 \mathrm{~h}$ post/injection. Embryo grading was done by standard morphology assessment. Embryo transfer was done on day 3 following oocyte retrieval. Luteal phase support with $800 \mathrm{mg}$ of micronized progesterone (Prontogest, Marcyrl Co., Egypt) was initiated on the same day of oocyte retrieval. One cycle only was made for each patient in both groups.

Pregnancy was assessed by serum hCG assay after 15 days from embryo transfer and then confirmed when a gestational sac with positive fetal pulsation was visualized at the vaginal US after two further weeks later. Only cases with US confirmation of pregnancy were counted in the calculation of pregnancy and implantation rates, whereas biochemical pregnancies were not considered.

\section{Outcome measures}

The primary outcome measure was the clinical pregnancy rate per ET cycle. The clinical pregnancy was defined as the presence of a gestational sac with the detection of fetal heartbeat detection at 6-7 weeks of gestation.

Secondary outcome measures were the live birth rate, the occurrence of OHSS, total dose of gonadotropins, E2 concentration on the day of hCG administration, cycle cancellation rate, number of cumulus oocyte complexes (COCs) retrieved, number of metaphase I and II oocytes, and fertilization rates, embryo grade classified as grade $(1,2,3)$.

\section{Statistical analysis and sample size justification}

A sample size calculation was done to calculate the number of subjects needed in each group. Reference to
Rimington et al. [16], the pregnancy rate in LOD and IVF was $36 \%$. We assumed that our current pregnancy rate would be $50 \%$, with a significance level of 0.05 and $80 \%$ power, at least 190 patients (95 patients per group). A total sample size of 200 was required to consider any dropouts.

All statistical tests were done using a significance level of $95 \%$. A value for $p<0.05$ was considered statistically significant. SPSS software (Statistical Package for the Social Sciences, version 20.0, SSPS Inc., Chicago, IL, USA) was used for the statistical analyses. Data were presented as (mean \pm SD) or median (range) for continuous variables and as frequency and percent for categorical variables. Comparisons between groups were done using Phi-Cramer test for categorical variables. The study adhered to the CONSORT guideline.

\section{Results}

All PCOS subjects resistant to CC (285) who came to the center and were willing to do ICSI were asked to participate in the study. Forty-six subjects refused to participate, and 27 subjects were excluded; 15 were not fit for surgery and 12 could not convince the husband to do ICSI. Enrolled subjects (212) were randomized to the drilling group and the control group, 106 in each group. Twelve subjects were excluded after randomization where ET was canceled in 5/106 cases in the drilling group (three cases because of no fertilization due to unexpected bad ejaculation and two cases because of no good cleavage) while 7/106 cases were canceled in the control group (three cases because of severe OHSS, two cases because of no fertilization, and two cases because of no good cleavage). The dispositions of these subjects are shown (Fig. 1).

\section{Baseline characteristics}

Only 200 (who completed the ICSI-ET cycle) subjects were included in the analysis, 101 in the LOD group, and 99 in the control group. There was no statistically significant difference $(p>0.05)$ between the two groups regarding the age, duration of infertility, and BMI, as shown in Table 1.

The two groups were comparable $(p>0.05)$ regarding the AFC, AMH level, FSH level, LH level, the estradiol (E2) level, the prolactin (PRL) level, and the thyroidstimulating hormone (TSH), as shown in Table 1.

\section{Ovarian induction and ICSI parameters}

Ovarian stimulation days were significantly $(p<0.001)$ higher in the drilling group 12.91 (1.91) days than in the control group 11.24 (2.03) days. Also, the dose of gonadotropin was insignificantly ( $p$ value 0.117 ) higher in the drilling group 36.42 (11.23) ampoules than in the control group 34.09 (9.67) ampoules. Also, there 


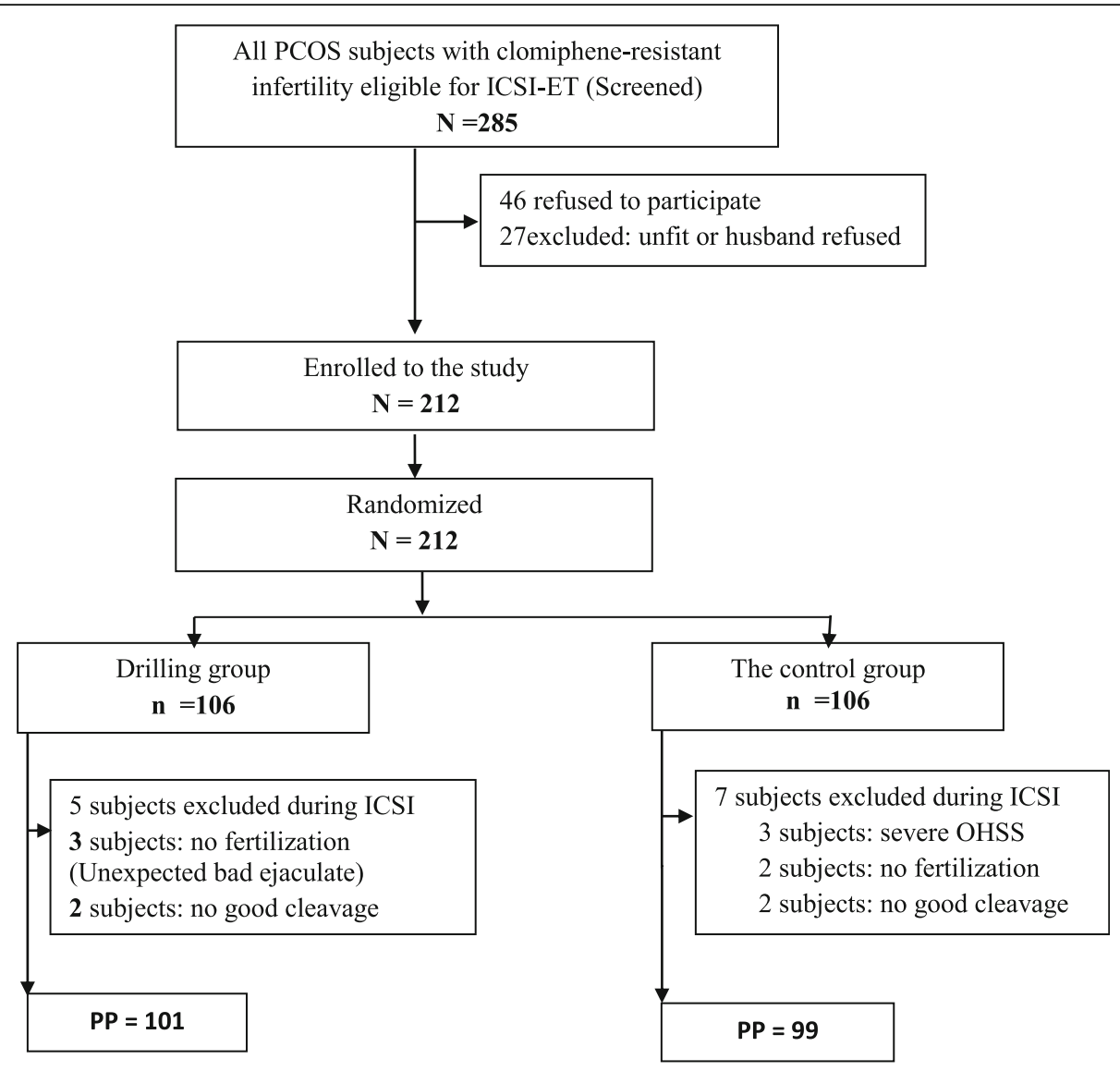

Fig. 1 Consort diagram

was a significant $(p<0.001)$ difference in the triggering day between the two groups which was slightly later in the drilling group. In addition, there was a significant $(p<0.001)$ difference in the dosage protocol between both groups, with lower fixed dosage protocol (24\%) of patients in the drilling group compared to (67\%) the control group.
Endometrium thickness, E2 level at triggering day, and the number of oocytes retrieved were significantly $(p<$ 0.001) lower in the drilling group than in the control group, as shown in Table 2.

In spite of the number of embryos cleaved, the numbers of grade 1 and grade 2 embryos were significantly $(p<0.01)$ different between both groups; the number of

Table 1 Baseline characteristics

\begin{tabular}{|c|c|c|c|}
\hline & $\begin{array}{l}\text { Drilling group } \\
N=101\end{array}$ & $\begin{array}{l}\text { Control group } \\
N=99\end{array}$ & $p$ \\
\hline Age in years & $30.4(4.4)$ & $29.1(5.9)$ & 0.067 \\
\hline Duration of infertility in years & $6.6(2.8)$ & $6.5(3.6)$ & 0.8 \\
\hline $\mathrm{BMI}$ & $28.5(3.8)$ & $28.2(4.2)$ & 0.5 \\
\hline Antral follicle count (AFC) before drilling & $16.5(4.9)$ & $16.5(5.2)$ & 0.9 \\
\hline Anti-Mullerian hormone (AMH) in $\mathrm{ng} / \mathrm{ml}$ before drilling & $2.7(0.6)$ & $2.8(0.8)$ & 0.4 \\
\hline Follicle stimulating hormone $(\mathrm{FSH})$ in $\mathrm{mlU} / \mathrm{ml}$ & $6.0(1.5)$ & $5.9(1.6)$ & 0.5 \\
\hline Luteinizing hormone $(\mathrm{LH})$ in $\mathrm{mlU} / \mathrm{ml}$ & $9.0(1.6)$ & $9.1(2.5)$ & 0.8 \\
\hline Estradiol (E2) in $\mathrm{pg} / \mathrm{ml}$ & $54.1(20.2)$ & $54.0(21.1)$ & 0.9 \\
\hline Prolactin (PRL) in $\mathrm{ng} / \mathrm{ml}$ & $12.5(7.6)$ & $12.43(5.1)$ & 0.9 \\
\hline Thyroid stimulating hormone (TSH) & $1.9(0.8)$ & $1.88(0.8)$ & 0.9 \\
\hline
\end{tabular}

Data are presented as mean (SD) 
Table 2 Ovarian induction and ICSI parameters

\begin{tabular}{|c|c|c|c|}
\hline & $\begin{array}{l}\text { Drilling group } \\
N=101\end{array}$ & $\begin{array}{l}\text { Control } \\
N=99\end{array}$ & $p$ \\
\hline Dose of gonadotropin (number of ampoules) & $36.4(11.2)$ & $34.1(9.7)$ & 0.1 \\
\hline Days of gonadotropin injection (stimulation days) & $12.9(1.9)$ & $11.2(2.0)$ & $<0.001$ \\
\hline Triggering day (day of HCG injection) & $14.4(2.0)$ & $13.2(2.0)$ & $<0.001$ \\
\hline \multicolumn{4}{|l|}{ Dosage protocol, number (\%) } \\
\hline Fixed & $24(24)$ & $66(67)$ & \multirow[t]{3}{*}{$<0.001$} \\
\hline Step up & $77(76)$ & $25(25)$ & \\
\hline Step down & $0(0)$ & $8(8)$ & \\
\hline Endometrium thickness in $\mathrm{mm}$ & $9.5(1.77)$ & $11.8(1.9)$ & $<0.001$ \\
\hline Estradiol (E2) level at triggering day & $2371.9(898.51)$ & $3168.1(1541.9)$ & $<0.001$ \\
\hline Number of oocytes retrieved & $10.7(6.05)$ & $13.6(5.5)$ & $<0.001$ \\
\hline Mature oocytes (M2) & $7.4(3.69)$ & $8.4(3.3)$ & 0.0499 \\
\hline Less mature oocytes (M1) & $1.7(1.54)$ & $2.6(1.6)$ & $<0.001$ \\
\hline Germinal vesicles (GV) or immature oocytes & $1.4(1.96)$ & $2.1(2.2)$ & 0.014 \\
\hline Empty zona (EZ) & $0.3(0.85)$ & $0.1(0.7)$ & 0.1 \\
\hline Number of fertilized oocytes & $6.2(3.09)$ & $6.3(2.5)$ & 0.6 \\
\hline AFC after LOD & $12.4(3.4)$ & $16.5(5.2)$ & $<0.001$ \\
\hline AMH after LOD & $2.1(1.3)$ & $2.8(0.8)$ & $<0.001$ \\
\hline Number of embryos cleaved & $5.4(2.8)$ & $4.3(2.0)$ & 0.002 \\
\hline Grade 1 embryos & $4.3(2.3)$ & $3.0(1.3)$ & $<0.001$ \\
\hline Grade 2 embryos & $0.7(0.9)$ & $1.2(1.0)$ & 0.001 \\
\hline Grade 3 embryos & $0.3(0.6)$ & $0.3(0.5)$ & 0.8 \\
\hline Number of embryos transferred & $3.3(1.0)$ & $3.1(1.5)$ & 0.3 \\
\hline Day of embryo transfer (usually day 3 ) & $3.0(0.4)$ & $2.7(1.0)$ & 0.049 \\
\hline Number of embryos ready for freezing & $1.2(1.9)$ & $0.8(1.6)$ & 0.1 \\
\hline
\end{tabular}

All data are presented as mean (SD) except dose protocol

embryos transferred and the number of frozen transferred were not significantly different ( $p$ value 0.324$)$, as shown in Table 2.

\section{Implantation rate and pregnancy outcomes}

The implantation rate was non-significantly $(p$ value $=$ 0.3) higher in the drilling group than in the control group. It was $17.8 \%$ in the drilling group and $14.5 \%$ in the control group.

The clinical pregnancy rate per ET cycle, as confirmed by US, was significantly ( $p=0.046)$ higher in the drilling group than in the control group. It was $51 \%$ in the drilling group and $36 \%$ in the control group.

The number needed to harm (NNH) equals 6.61; only one out of seven cases using drilling will not get pregnant.

In addition, multiple pregnancies were not significantly $(p=0.265)$ different between both groups, as shown in Table 3.

Abortion rate till 20 weeks was 5 cases $(9.6 \%)$ of pregnant women in the drilling group and 4 cases $(10.8 \%)$ of pregnant women in the control group ( $p$ value $=0.6)$.
The live birth rate was $42(41 \%)$ and 29 (29\%) in the drilling group and the control group, respectively ( $p$ value $=0.075)$.

\section{Safety outcomes}

There was a significant $(p=0.046)$ difference as regards the rate of OHSS between the two groups. OHSS was more in the control group (23\%) than in the drilling group (11\%). Severe OHSS was occurred only in the control group (7\%), as shown in Table 3.

In addition, coasting was significantly needed $(p<0.001)$ to stop the stimulation for fear of OHSS in the control group (18\%) as compared to the drilling group (only $2 \%$ ).

Moreover, no major complications were encountered following LOD. Minor complications encountered were pain, minimal vaginal bleeding, and postoperative cough in some cases.

\section{Discussion}

In our study, we tested the question: Does LOD, when used in PCOS patients with clomiphene-resistant infertility, have 
Table 3 Pregnancy outcomes and OHSS

\begin{tabular}{|c|c|c|c|}
\hline & Drilling group & Control group & $p$ \\
\hline \multicolumn{4}{|c|}{ Pregnancy rate: confirmed by HCG then US } \\
\hline Yes & $52(51 \%)$ & $35(36 \%)$ & \multirow[t]{2}{*}{0.044} \\
\hline No & $49(49 \%)$ & $64(64 \%)$ & \\
\hline EER (experimental event rate) & \multicolumn{3}{|l|}{0.51} \\
\hline CER (control event rate) & \multicolumn{3}{|l|}{0.36} \\
\hline ARR (absolute risk reduction) & \multicolumn{3}{|c|}{$-0.15(-0.28$ to -0.02$)$} \\
\hline NNT (number needed to treat) & \multicolumn{3}{|c|}{$-6.61(-3.65$ to -65.46$)$} \\
\hline NNH (number needed to harm) & \multicolumn{3}{|c|}{6.61 (3.65 to 65.46) } \\
\hline \multicolumn{4}{|l|}{ Pregnancy outcome } \\
\hline Single & $46(45.5 \%)$ & $32(32.3 \%)$ & 0.3 \\
\hline Twins & $5(5.0 \%)$ & $2(2.0 \%)$ & \\
\hline Triplets & $1(1.0 \%)$ & $1(1.0 \%)$ & \\
\hline Implantation rate & $17.8 \%$ & $14.5 \%$ & 0.3 \\
\hline Abortion rate till 20 weeks & $5(9.6 \%)$ & $4(10.8 \%)$ & 0.6 \\
\hline Live birth rate & $42(41 \%)$ & $29(29 \%)$ & 0.075 \\
\hline \multicolumn{4}{|l|}{ OHSS } \\
\hline No & $90(89 \%)$ & $76(77 \%)$ & 0.046 \\
\hline Mild & $8(8 \%)$ & $3(3 \%)$ & \\
\hline Moderate & $3(3 \%)$ & $13(13 \%)$ & \\
\hline Severe & $0(0 \%)$ & $7(7 \%)$ & \\
\hline \multicolumn{4}{|l|}{$\begin{array}{l}\text { Coasting: to stop stimulation } \\
\text { for fear of OHSS }\end{array}$} \\
\hline No coasting & 99 (98\%) & 81 (82\%) & $<0.001$ \\
\hline Coasting & $2(2 \%)$ & 18 (18\%) & \\
\hline
\end{tabular}

Data are presented as number (\%)

a positive impact on outcomes of ICSI-ET in terms of increased pregnancy rate? The results of this study showed that LOD may be effective in increasing the pregnancy rate in those patients. The live birth rate was insignificantly higher in the drilling group than in the control group.

The results of our study showed that the ovarian stimulation days were significantly higher in the drilling group. Also, the dose of gonadotropin was insignificantly higher in the drilling group. Also, the triggering day was significantly later in the drilling group. The fixed dosage protocol was used in a less number of patients in the drilling group than in the control group. Endometrium thickness, E2 level at triggering day, and the number of oocytes retrieved were significantly lower in the drilling group. In spite of the number of embryos cleaved, the numbers of grade 1 and grade 2 embryos were significantly different between both groups; the number of embryos transferred and the number of frozen transferred were not significantly different. Drilling significantly improved the pregnancy rate decreased the rate of OHSS and reduced the need for coasting. LOD did not improve the oocyte number; however, the quality of the oocytes might be improved. One of the disadvantages of LOD in our study is the reduction of the ovarian reserve that expressed in decreasing the number of oocytes retrieved and increasing the stimulation days and the dose; however, LOD seems to normalize hyper-responder patients with improvement in the oocytes' quality, not the number. Also, we have an increased rate of multiple pregnancies, but this is simply due to the increase of the number of embryos transferred, and it is accepted for our patients as they accept the risk of multiple pregnancies more than the failure of ICSI cycles.

Improved oocyte quality in the LOD group may be due to drilling itself or secondarily to decrease the need to coasting and step down gonadotropin therapy.

To our knowledge, during the last two decades, there were only three studies addressed in the same topic; two of them were retrospective studies $[14,15]$ and only one small sample size randomized controlled trial [16] which is also mentioned in the Cochrane review [17].

Contrary to the results of our study, all of the three studies showed that there were no significant increases in pregnancy rates due to LOD. However, all mentioned increased trend but it was not statistically significant. In addition, as mentioned before, two of these studies were observational retrospective studies and only one RCT, but with a small sample size of 50 women.

The results of Rimington et al. [16] showed no evidence of a significant difference in pregnancy rate with the addition of LOD to IVF when compared to IVF alone. Also, it showed no evidence of a significant difference neither in multiple pregnancy rates nor in the OHSS rate.

The retrospective study of Tozer et al. [15] showed no statistically significant differences $(p>0.05)$ were observed between both groups as regards the number of retrieved oocytes, ongoing pregnancy rates, and the incidence of OHSS. However, the number of embryos available for transfer was significantly higher in the control group (without drilling).

The results of the retrospective study of Eftekhar et al. [14] showed that ovarian cauterization before ICSI-ET in patients with PCOS reduced the risk of OHSS. Despite the same pregnancy rate in both groups, more obtained oocytes and embryos were seen on women without ovarian drilling than women with LOD.

The advantages of this study are being assessorblinded multicenter randomized controlled trial with enough sample size. Moreover, it does offer the benefit of being the first head-to-head comparison using a randomized controlled trial design. However, one limitation of this study is that the drilling group needs more gonadotropins dose and more stimulation days. Another limitation of the study is that a higher percentage (30\%) of the original population declined/excluded from the 
study $(85 / 285)$ which may jeopardize the generalizability of the study. Moreover, we did not test for glucose metabolism in such population with insulin resistance. Another limitation was missing reporting the details of coasting; however, we did it in a range of 1-2 days. Also, the exact cause of the cancellation was not reported in every case, which is another limitation in the current study.

\section{Conclusions}

Finally, we conclude that the laparoscopic ovarian drilling for PCOS patients with clomiphene-resistant infertility before ICSI-ET improves the clinical pregnancy outcome with an OHSS rate reduction.

\section{Abbreviations \\ AFC: Antral follicle count; AMH: Anti-Mullerian hormone; ARR: Absolute risk reduction; ASRM: American Society for Reproductive Medicine; BMI: Body mass index; CC: Clomiphene citrate; Cl: Confidence interval; COCs: Cumulus- oocyte complexes; ESHRE: European Society of Human Reproduction and Embryology; ICSI-ET: Intra-Cytoplasmic Sperm Injection/Embryo Transfer; IVF: In vitro fertilization; LOD: Laparoscopic ovarian drilling; NNH: Number needed to harm; OHSS: Ovarian hyper-stimulation syndrome; PCOS: Polycystic ovarian syndrome; RCT: Randomized controlled trial; SD: Standard deviation; SERMs: Selective estrogen receptor modulators}

\section{Acknowledgements}

We would like to acknowledge Dr. Emad R Issak (ClinAmygate) for his efforts in the statistical analysis and editing of this manuscript.

\section{Authors' contributions}

AMN is the principal investigator of the study, study design, management, supervision, and final revision. HA, HN, and RAM contributed to the study design, patients' recruitment, and manuscript revision. All authors read and approved the final manuscript.

\section{Funding}

This research did not receive any specific grant from any funding agency in the public, commercial, or not-for-profit sector.

\section{Availability of data and materials}

The datasets used and/or analyzed during the current study are available from the corresponding author on reasonable request.

\section{Ethics approval and consent to participate}

The study was approved by the medical ethical review committee of Cairo University on December 14, 2014, with registration number no: OG-5-14-12 2014. The purpose of this study was clearly explained in the Arabic language to all subjects before their enrollment to the study, and an informed consent form was signed by and obtained from all of those enrolled.

\section{Consent for publication}

Not applicable

\section{Competing interests}

The authors declare that they have no competing interests.

Received: 11 October 2019 Accepted: 10 February 2020

Published online: 03 April 2020

\section{References}

1. Asgharnia M, Mirblook F, Ahmad SM (2011) The prevalence of polycystic ovary syndrome (PCOS) in high school students in Rasht in 2009 according to NIH criteria. Int J Fertil Steril. 4:156-159

2. Boomsma CM, Eijkemans MJ, Hughes EG, Visser GH, Fauser BC, Macklon NS (2006) A meta-analysis of pregnancy outcomes in women with polycystic ovary syndrome. Hum Reprod Update. 12(6):673-683
3. Kawwass JFLT, Berga SL (2010) An algorithm for treatment of infertile women with polycystic ovary syndrome. Middle East Fertil Soc J. 15(4):231-239

4. Rebecca LR, Goldberg JM (2011) Laparoscopic ovarian drilling for clomiphene-resistant polycystic ovary syndrome. Semin Reprod Med. 29(2): 138-146

5. Seow KM, Juan CC, Hwang JL, Ho LT (2010) Laparoscopic surgery in polycystic ovary syndrome: reproductive and metabolic effects. Semin Reprod Med. 26(1):101-110

6. Sirmans SM, Pate KA (2014) Epidemiology, diagnosis, and management of polycystic ovary syndrome. Clin Epidemiol 6:1-13

7. Badawy A, Elnashar A (2011) Treatment options for polycystic ovary syndrome. Int J Womens Health. 3:25-35

8. Imani B, Eijkemans MJ, te Velde ER, Habbema JD, Fauser BC (1998) Predictors of patients remaining anovulatory during clomiphene citrate induction of ovulation in normogonadotropic oligoamenorrheic infertility. J Clin Endocrinol Metab. 83(7):2361-2365

9. Ferraretti $A P$, Gianaroli L, Magli MC, Lammarrone E, Feliciani E, Fortini D (2001) Transvaginal ovarian drilling: a new surgical treatment for improving the clinical outcome of assisted reproductive technologies in patient with polycystic ovary syndrome. Fertil Steril. 76(4):812-816

10. Gjonnaess H (1984) Polycystic ovarian syndrome treated by ovarian electrocautery through the laparoscope. Fertil Steril. 41(1):20-25

11. Amer SA, Li TC, Cooke ID (2002) Laparoscopic ovarian diathermy in women with polycystic ovarian syndrome: a retrospective study on the influence of the amount of energy used on the outcome. Hum Reprod. 17(4):1046-1051

12. Thessaloniki ESHRE/ASRM-Sponsored PCOS Consensus Workshop Group (2008) Consensus on infertility treatment related to polycystic ovary syndrome. Fertil Steril 89(3):505-522

13. Heijnen EM, Eijkemans MJ, Hughes EG, Laven JS, Macklon NS, Fauser BC (2006) A meta-analysis of outcomes of conventional IVF in women with polycystic ovary syndrome. Hum Reprod Update. 12(1):13-21

14. Eftekhar M, Dehghani Firoozabadi R, Khani P, Ziaei Bideh E, Forghani H (2016) Effect of laparoscopic ovarian drilling on outcomes of in vitro fertilization in clomiphene-resistant women with polycystic ovary syndrome. Int J Fertil Steril. 10(1):42-47

15. Tozer AJ, Al-Shawaf T, Zosmer A, Hussain S, Wilson C, Lower AM et al (2001) Does laparoscopic ovarian diathermy affect the outcome of IVF-embryo transfer in women with polycystic ovarian syndrome? A retrospective comparative study. Hum Reprod. 16(1):91-95

16. Rimington MR, Walker SM, Shaw RW (1997) The use of laparoscopic ovarian electrocautery in preventing cancellation of in vitro fertilization treatment cycles due to risk of ovarian hyperstimulation syndrome in women with polycystic ovaries. Hum Reprod. 12(7):1443-1447

17. Farquhar C, Brown J, Marjoribanks J (2012) Laparoscopic drilling by diathermy or laser for ovulation induction in anovulatory polycystic ovary syndrome. Cochrane Database Syst Rev (6):CD001122. https://doi.org/10. 1002/14651858.CD001122

\section{Publisher's Note}

Springer Nature remains neutral with regard to jurisdictional claims in published maps and institutional affiliations.

\section{Submit your manuscript to a SpringerOpen ${ }^{\circ}$ journal and benefit from:}

- Convenient online submission

- Rigorous peer review

- Open access: articles freely available online

- High visibility within the field

- Retaining the copyright to your article

Submit your next manuscript at $>$ springeropen.com 